\title{
Do obese patients with primary knee osteoarthritis benefit from a single bout of moderate intensity aerobic exercise?
}

\author{
Reham M. Shaat ${ }^{1 *}$, Tamer O. Elsaid ${ }^{1}$, Aymen EL Baz ${ }^{2}$, Rehan Monir ${ }^{2,3}$ and Shereen Aly Machaly ${ }^{1}$
}

\begin{abstract}
Background: Obesity and osteoarthritis are often concomitant. Both are accompanied by oxidative stress and stimulated inflammatory response. Exercise is considered a substantial treatment in rehabilitation of both conditions. Yet most of literature reported the benefits of regular exercising, while there is paucity about the effects of single low to moderate exercise session. This study aimed to investigate the possible potential effects of a single bout of moderate exercise in obese middle-aged individuals with knee osteoarthritis (KOA).

Results: Score of pain and Western Ontario and Mc-Master University (WOMAC) had been improved $24 \mathrm{~h}$ after exercise. Glutathione reductase (GR) activity increased post-exercise but decreased the next $24 \mathrm{~h}$ though remained higher than baseline. Blood glucose level was reduced post-exercise whereas blood lipids still uninfluenced by exercise. Markers of inflammation remained unchanged after exercise session.

Conclusion: A single bout moderate-intensity aerobic exercise is effective in improving pain and antioxidant activity among middle-aged obese patients with primary knee OA. Single bout of mild to moderate exercise may help in treatment of obese patients with KOA.
\end{abstract}

Trial registration: Clinical Trials.gov Identifier: NCT03927339

Keywords: Obesity, Knee osteoarthritis, Single aerobic exercise session, Glutathione reductase, Oxidative stress

\section{Background}

Dramatically, worldwide obesity incidence has increased throughout the recent decades. Obesity and its accompanying disorders like KOA now represent significant health impacts in both developed and developing countries [1].

$\mathrm{KOA}$ is a clinical multifactorial disorder with several etiologies and different risk factors that probably contribute to its manifestations, and of them is obesity and inflammation, the mostly blamed and constitute the highest associated disorders with the disease [2].

Increased oxidative stress seems to be an important component of both obesity and osteoarthritis (OA) and

\footnotetext{
*Correspondence: rehamshaat@mans.edu.eg; reham1975@yahoo.com; rehamshaat@gmail.com

${ }^{1}$ Faculty of Medicine, Department of Rheumatology and Rehabilitation, Mansoura University, Elgomhoria St, Mansoura, Egypt

Full list of author information is available at the end of the article
}

may formulate an important track joining obesity with the elevated incidence of OA. In obesity, as well as in $\mathrm{OA}$, the cellular antioxidant capability is insufficient for detoxifying the reactive oxygen species (ROS), with the resulting oxidative stress that may cause damage of cellular enzymes and organelles, resulting in the development of insulin resistance, degrading components located extracellular such as collagens and proteoglycans, and additionally, increasing the susceptibility of chondrocytes to oxidant-mediated cell death [3].

Currently, one of the most studied antioxidants is glutathione, probably due to its endogenous synthesis throughout the body and being basically found in all cells, and plays any roles in which it is involved [4].

Previous studies have reported glutathione reductase (GR) changes seen in serum, the enzyme that is responsible for the restoration of reduced glutathione from

\section{Springer Open}

() The Author(s). 2020 Open Access This article is licensed under a Creative Commons Attribution 4.0 International License, which permits use, sharing, adaptation, distribution and reproduction in any medium or format, as long as you give appropriate credit to the original author(s) and the source, provide a link to the Creative Commons licence, and indicate if changes were made. The images or other third party material in this article are included in the article's Creative Commons licence, unless indicated otherwise in a credit line to the material. If material is not included in the article's Creative Commons licence and your intended use is not permitted by statutory regulation or exceeds the permitted use, you will need to obtain permission directly from the copyright holder. To view a copy of this licence, visit http://creativecommons.org/licenses/by/4.0/. 
oxidized glutathione via the application of nicotinamide adenine dinucleotide (NADH) [5, 6].

Being an interventional strategy against obesity and related disorders, the advocacy of exercise is gaining more added significance. Additionally, numerous studies revealed that physical and exercise activities alone have several metabolic benefits such as improved tissue sensitivity to insulin and elevated peak oxygen consumption [7].

Increased production of ROS due to exercise can be beneficial by inducing particular adaptations, such as increased resistance to oxidative stress, increased antioxidant/oxidative damage repairing enzyme activity, and lower levels of oxidative damage [8].

Available data supports the impact of life long exercise training on the level of pain and the anti-inflammatory status in KOA patients. However, exercise might produce a short-term pro-inflammatory response depending on physical training of the patient, mode, severity, and duration of the exercise [9-11].

An increased understanding of the molecular basis for exercise-induced metabolic effects is important in developing optimal exercise interventions for primary KOA. Since many of KOA are usually sedentary and may not be able to practice exercise regularly and lack of studies revealing the effect of single session of exercise on KOA, this raises the question "can they benefit from sporadic single bouts of mild to moderate exercise?"

This study aimed to determine how mild to moderate exercise influences metabolic, antioxidant, and inflammatory responses of untrained obese middle-aged patients with KOA.

\section{Methods}

\section{Study population}

This is an observational study among a cohort of sedentary Egyptian patients that included thirty-four obese patients diagnosed with primary KOA according to the criteria of the American College of Rheumatology (ACR) [12] and were selected from those attending the outpatient clinics of Rheumatology and Rehabilitation Department, Mansoura University from June 2019 to October 2019 (28 females and 6 males; mean age, $46.94 \pm 10.84$ years; body mass index, 40.91 $\pm 10.09 \mathrm{~kg} / \mathrm{m}^{2}$ ). In addition, 30 healthy volunteers without clinical and radiological manifestations of KOA attending in the hospital for regular check-up (22 females and 8 males; mean age, $50.7 \pm 10.5$; BMI $24.3 \pm 1.3)$ participated in the study.

This study was approved by the Institutional Research Board of Faculty of Medicine, Mansoura University code R.18.04.137. A written informed consent was obtained from all participants before the start of the study.

\section{Inclusion criteria}

The inclusion criteria are as follows: KOA with obesity, age ranging from 36 to 56 years old, sedentary with no previous training, ability to understand and follow commands, and ability to walk independently.

\section{Exclusion criteria}

The exclusion criteria are as follows: any conditions that medically contraindicated for moderate aerobic exercise (as detected by prescreening questions, i.e., cardiac or coronary artery disease as ischemic cardiomyopathy, chronic lung disease, asthma, uncontrolled hypertension), inability to exercise via treadmill (i.e., extra neuromuscular or lower extremity conditions or any other medical contraindication that could prevent them from walking on treadmill as disorders impacting hip movement or gait, history of previous hip or knee surgery, peri-articular fractures, or lower limb injuries), history of recent joint infection or acute joint inflammation, any recent history of intraarticular joint injection (hyaluronic acid, steroids, etc.) during the previous 6 weeks of study, intake of antioxidant supplementation, and the use of psychotropic agents. All secondary causes of KOA were excluded (gout, pseudogut, trauma, and autoimmune diseases).

Patients who did not sign the consent (4 patients), did not attend the next day for blood sample (3 patients), and/or did not complete the session due to exhaustion (5 patients) were also excluded.

\section{Data collection}

Collection of basic clinical data was done through interviewing the participants that included demographic characteristics, history of associated comorbidities such as diabetes and hypertension, history of previous knee or hip surgery, and history of drug intake or recent joint injection.

\section{Evaluation of $O A$}

The Western Ontario and Mc-Master University (WOMAC) OA scoring index was used for the evaluation of joint pain, stiffness, and function in OA patients [13]. Total WOMAC is the sum of three subscales scores. High WOMAC score indicates more pain and stiffness and severe functional limitation.

\section{Radiological assessment}

Bilateral antero-posterior (AP) standing knee radiographs were done to confirm the presence of OA and its degree. Grading of X-rays was done by Kellgren and Lawrence (K/L) scale for KOA severity [14]. Subjects with radiographic KOA of $\mathrm{KL}$ grade $\geq 2$ in one knee were enrolled. The worst affected knee grading of each patient was used for data analysis. 


\section{Treadmill training protocol}

Each eligible participant in the patients' group was then prospectively assigned to undergo exercise session of full-body-weight treadmill training. Once the participants were prepared, muscle stretching for $5 \mathrm{~min}$ and slow walking for 3 min were performed as a warm-up exercise before starting the training. Stretching was performed similarly to all the participants to quadriceps femoris and the hamstring. During warm-up period, it was enforced for $3 \mathrm{~min}$ at the speed of $1 \mathrm{~km} / \mathrm{h}$. Then, patients began the walking trials and walked at 1 to 1.34 $\mathrm{m} / \mathrm{s}$ on an electric treadmill (JACO fitness: JACO-212C) (South Korea).

\section{Exercise intensity}

In this study, exercise intensity was determined through applying Metabolic Equivalents (METs) and Rate of Perceived Exertion (RPE) as guidelines. Activity that generates energy expenditure of 3.0 to 6.0 (METs) was defined as moderate aerobic exercise [15] for the determination of exercise intensity, time, and frequency; it was set at low to moderate, which is between 40 and $60 \%$ of the maximum heart rate, rating of RPE from 12 to 14 . In this study, moderate aerobic exercise was also defined by a participant's subjective Rate of Perceived Exertion (RPE) of 4-6 on a 10-point scale. Additionally, the 15-point Borg Rating of Perceived exertion scale has been introduced to each participant and instructed in its use. The RPE scale was easily visible to the participants on the wall in front of the treadmill. Instructions regarding exercise and measurements of associated METs were determined based upon earlier studies which proved that walking on a level surface at 3.0 MPH is equal to 3.3 METs, and walking at 4.5 MPH generates 6.3 METs [16]. Participants walked at a pace of 2.5 to $3.0 \mathrm{MPH}$ on a treadmill with zero incline for $30 \mathrm{~min}$, and they were instructed to walk at a pace that neither caused pain nor increased symptoms [17]. To minimize pain that may occur due to treadmill exercise, 5-min rest was allowed after $15 \mathrm{~min}$ of exercise. In the control group, the subjects remained at rest.

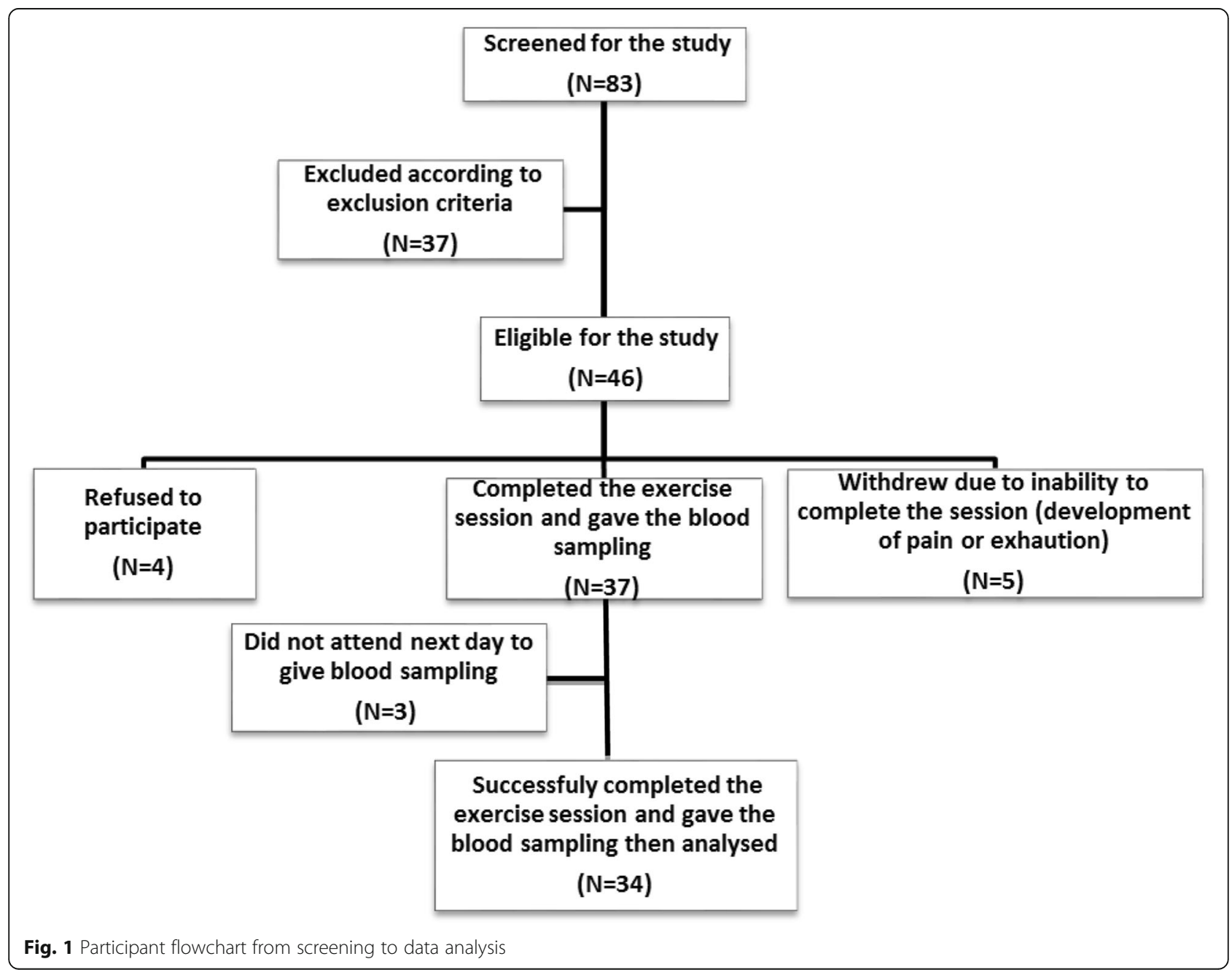




\section{Laboratory assays}

Blood samples were drawn before and after exercise (within $0.5 \mathrm{~h}$ after exercise and at $24 \mathrm{~h}$ ). The whole biochemical measurements were carried out on frozen plasma samples obtained throughout centrifugation of freshly drawn blood $\left(3000 \times g\right.$ for $20 \mathrm{~min}$ at $4{ }^{\circ} \mathrm{C}$ ) and stored subsequently at $-70{ }^{\circ} \mathrm{C}$. Blood lipid profiles, including total cholesterol (TC), HDL cholesterol (HDLC), calculated LDL cholesterol (LDL-C), and triglycerides (TG) concentrations, were determined by enzymatic assays, as well as fasting blood glucose level.

\section{IL-6, CRP}

IL-6 serum concentrations were determined using sandwich high sensitivity ELISA kit for quantitative detection of human IL-6 according to manufacturer's protocol (Boster Immuno leader by Boster Biological Technology Co. Inc.). Detection of serum CRP was performed by Solid Phase Sandwich ELISA according to manufacturer's instructions (Quantikine Human CRP Immunoassay, R\&D systems).

\section{Glutathione reductase assay}

Spectro-photometric determination of GR activity in the serum was measured as described by Calberg and Mannervic [18] using commercially available Glutathione Reductase Assay Kit (Sigma Chemical Company, St Louis, MO, USA). Reduction of glutathione disulfide (GSSG) was catalyzed by glutathione reductase throughout the oxidation of reduced form of nicotinamide adenine dinucleotide phosphate (NADPH) to nicotinamide adenine dinucleotide phosphate (NADP+). The decrease in absorbance was measured at 340 .

\section{Statistical analysis}

Data were analyzed using the SPSS software version 21.0. Mean and standard deviation (mean \pm SD) was used to represent data. Kolmoggorov-Smirnov test was used to determine the homogeneity of data distribution. Changes of pain and laboratory parameters among participants before and after exercise and $24 \mathrm{~h}$ after were assessed using ANOVA. Means of continuous variables before and after physical exercise were compared by paired Student's $t$ test. Pearson's correlation test was performed to assess clinical and laboratory parameters correlation. A $p$ value $\leq 0.05$ was considered statistically significant.

\section{Results}

\section{Baseline characteristics}

A total of 34 obese KOA patients shared in and completed this study with average age $46.94 \pm 10.84$ years (range 30-60 years). Eighty-two percent of the participants were females, and average body was mass index
$40.91 \pm 10.09 \mathrm{~kg} / \mathrm{m}^{2}$. The flow diagram of the patients participated in this study is shown in (Fig. 1). The demographic characteristics, clinical, and laboratory results of the study participants are listed in Table 1.

GR activity was significantly lower, and the inflammatory markers were significantly higher in patients at baseline than controls. Significant differences were also found between patients at baseline and controls as regards lipid profile and blood glucose level (Table 1)

\section{After exercise}

The patients did not report significant increase in pain post-exercise; furthermore, perceived pain was significantly diminished the next day (Tables 2 and 3) with median change of $20 \%$ from the baseline. In terms of functional status, a significant improvement in WOMAC score was noticed after $24 \mathrm{~h}$ with median change of $4.4 \%$ from baseline (Table 4).

Regarding the antioxidant activity, GR was significantly increased following exercise. Though this rise was shortlived and reduced later, it maintained higher levels than baseline for the next $24 \mathrm{~h}$, yet not significant (Table 5 ). While no change in IL-6 or CRP was demonstrated even instantly after exercise, blood lipid levels did not alter after exercise; meanwhile, blood glucose level was significantly reduced after exercise and remained lower than baseline level for the following day, although this difference turned insignificant (Table 6).

Table 1 Demographic, clinical, and laboratory data of the study participants

\begin{tabular}{llll}
\hline Characteristic & Patients & Control & $\boldsymbol{p}$ value \\
\hline Age (years) & $46.94 \pm 10.84$ & $50.7 \pm 10.5$ & 0.358 (NS) \\
Sex (male/female) & $6 / 28$ & $3 / 12$ & 0.85 \\
BMI (kg/m²) & $40.91 \pm 10.06$ & $24.3 \pm 1.3$ & $<0.001$ \\
VAS of pain & $5.07 \pm 1.16$ & NA & \\
WOMAC score & $70.26 \pm 11.21$ & NA & \\
KL score & $2.48 \pm 0.68$ & NA & \\
TC (mg/dL) & $210 \pm 22.17$ & $161.93 \pm 19.71$ & $<0.001$ \\
HDL-C (mg/dL) & $35.71 \pm 6.3$ & $43.87 \pm 6.17$ & $<0.001$ \\
LDL-C (mg/dL) & $149.71 \pm 23.34$ & $100.4 \pm 19.59$ & $<0.001$ \\
TG (mg/dL) & $123.86 \pm 17.4$ & $87.8 \pm 24.01$ & $<0.001$ \\
IL-6 (pg/mL) & $58.46 \pm 6.51$ & $46.78 \pm 1.61$ & $<0.001$ \\
Glucose level & $173.92 \pm 6.24$ & $101.76 \pm 17.07$ & $<0.001$ \\
CRP (mg/L) & $5.24 \pm 0.23$ & $3.33 \pm 0.32$ & 0.022 \\
GR activity (U/l) & $47.92 \pm 9.75$ & $59.13 \pm 12.53$ & 0.006
\end{tabular}

Data are expressed as mean values \pm standard deviation. The inter-group variability is determined by $t$ test. Significance level of $p$ value is $\leq 0.05$. Nonparametric parameters are shown as numbers, and the Chi square test $X^{2}$ was used to test their statistically significant differences

$B M I$ body mass index, VAS visual analogue scale, NA not applicable, $T C$ total cholesterol, $H D L$ high density lipoprotein, $L D L$ low density lipoprotein, $T G$ triglycerides, IL-6 interleukin-6, GR glutathione reductase 
Table 2 Pain score (VAS) before and after exercise

\begin{tabular}{lllll}
\hline VAS of pain & & & \\
\hline Pre-exercise baseline & Post-exercise & 24-h post-exercise & $\begin{array}{l}\text { \% change in score } \\
\text { median (range) }\end{array}$ & $F$ \\
\hline $5.07 \pm 1.16$ & $5.20 \pm 1.27$ & $4.47 \pm 1.30$ & $20(-25,33.3)$ & 13.45 \\
\hline
\end{tabular}

The intra-group comparison is shown before and after exercise. Data are expressed as mean values \pm standard deviation. Parameters failing normal distribution () are shown as median value and range (in brackets). The intra-group variability is determined by ANOVA test. Significance level of $p$ value is $\leq 0.05$

There was a significant negative correlation between level of GR at baseline and the percent change in its level after exercise $(r=-0.713, p=0.006)$. On the other hand, no other correlation was found between baseline GR activity and any of the clinical or laboratory parameters.

BMI correlated inversely with WOMAC score and positively with baseline IL-6 level $(r=-0.480,0.516$ and $p=0.044,0.017$ respectively). Age correlated as expected with $\mathrm{X}$-ray score $(r=0.482, p=0.027)$.

\section{Discussion}

Many studies have acknowledged the important effects of exercise in the treatment of knee OA and concomitant obesity with its related complications. Although acute strenuous and maintained exercise influences have been well investigated in OA patients, same information for single bout of low to moderate intensity is limited. Several studies had addressed the valuable benefits of exercises in improving pain and functional status of OA patients. Nevertheless, most of these studies evaluated the effects of repeated exercises for variable periods of time. Patients with moderate to severe KOA often obese, sedentary, and have functional disabilities preventing them from performing exercise on regular basis or being engaged in prolonged exercise programs. Increased-intensity or -duration exercises as well as regular training may not be feasible for these patients. We thought that single bouts of low- to moderateintensity exercise could have some benefits as midway between sedentariness and regular training in this particular category of patients [19].

Since obesity is considered an important co-morbidity of KOA, obese patients were chosen to participate in this study. Almost $65 \%$ of KOA diagnosed adults were either overweight or obese [20], and more than $30 \%$ of obese adults exhibit KOA [21]. Because women demonstrate higher rates of $\mathrm{KOA}$ incidence than men, the enrolled patients were mostly females [22] probably as a result of attenuated estrogens' protective effect after menopause and also their tendency to accumulate more fat than men [22]. The mean body mass index (BMI) of KOA female patients is relatively $24 \%$ greater than that of their healthy counterparts [23].

In the current study, we found that a single session of low- to moderate-intensity exercise for $30 \mathrm{~min}$ in obese untrained KOA elicited improvement in pain and WOMAC score after $24 \mathrm{~h}$ with significant increase in GR antioxidant activity after exercise. This increase remained more than the baseline for the next day though turned insignificant. Although earlier studies demonstrated that physical exercise is considered as oxidant stimulus that generating a ROS-dependent adaptive signaling [24, 25], this effect looks to be influenced by a high inter-individual variability [26]. Recently, studies revealed that oxidative stress induced by exercise is exacerbated particularly in obese subjects as a response to acute exercise [27] that subsequently induces an elevation in the endogenous antioxidant activity during acute exercise as a counterforce mechanism [28], which is in line with the current study results. For that, these findings suggest that, in certain conditions, even a short session of exercise may be adequate to activate antioxidant defense mechanisms against oxidative harm. In this context, this study speculates that when sedentary lifestyle is concomitant with severe obesity, an early antioxidant response may be needed to counteract the increased oxidative condition that happens following physical activity, whereas this does not happen in moderate obesity or sedentariness alone. Furthermore, a significant inverse relation was detected between GR baseline level and its percentage post-exercise change. When taking in consideration, the changes that occur in redox chemistry as a result of physical exercise, the capability of the body to sustain an optimum balance of glutathione is vitally important. The current study revealed an increase in levels of GR in response to mild non-exhaustive exercise as a body adaptive mechanism to effectively work against the

Table 3 Comparison between pain score at baseline, post-exercise, and $24 \mathrm{~h}$ after

\begin{tabular}{|c|c|c|c|c|c|c|c|c|}
\hline \multicolumn{9}{|l|}{ VAS of pain } \\
\hline \multirow{2}{*}{$\begin{array}{l}\text { \% change in score, } \\
\text { median (range) }\end{array}$} & \multicolumn{4}{|c|}{ Change between baseline and post-exercise } & \multicolumn{4}{|c|}{ Change between baseline and $24-h$ post-exercise } \\
\hline & Pre-exercise baseline & Post-exercise & $95 \% \mathrm{Cl}$ & $p$ value & Pre-exercise baseline & 24-h post-exercise & $95 \% \mathrm{Cl}$ & $p$ value \\
\hline $20(-25,33.3)$ & $5.07 \pm 1.16$ & $5.20 \pm 1.27$ & $(-0.53,0.24)$ & 0.44 & $5.07 \pm 1.16$ & $4.47 \pm 1.30$ & $(0.21,1.07)$ & 0.007 \\
\hline
\end{tabular}

The intra-group variability is determined by $t$ test. Significance level of $p$ value is $\leq 0.05$ 
Table 4 WOMAC score before and after exercise

\begin{tabular}{lllll}
\hline WOMAC score & & & \\
\hline $\begin{array}{l}\text { Pre-exercise } \\
\text { baseline }\end{array}$ & $\begin{array}{l}\text { 24-h post- } \\
\text { exercise }\end{array}$ & \% change in score & $95 \% \mathrm{Cl}$ & $p$ value \\
\hline $70.26 \pm 11.21$ & $67.78 \pm 12.78$ & $4.39 \pm 7.11$ & $0.62-5.38$ & 0.017
\end{tabular}

The intra-group comparison is shown before and after exercise. Data are expressed as mean values \pm standard deviation. The inter-group variability is determined by $t$ test. Significance level of $p$ value is $\leq 0.05$

increased free radicals that commonly occurs secondary to obesity and OA. Perhaps, this is the reason why the less the GR level in obese OA individuals, the more the increase in its release as a compensatory mechanism after exercise.

One of the potential hazards of exercise is that it might elicit a short-term mild to modest proinflammatory response that may aggravate the inciting state already exists in such kind of patients [29]. This happens particularly with acute intense exercise. In this terms, our study showed that moderate-intensity exercise did not provoke a significant inflammatory response on short-term in KOA patients. These results suggest that this kind of exercise does not provide enough stimulus that exacerbates inflammatory status of KOA.

This study assessed the influence of aerobic exercise on blood lipids before and after exercise, measured triglycerides and cholesterol as they are the most common lipids that cover 98 to $99 \%$ of all occurring natural lipids, and are widely taken as an index for confirming and detecting hyperlipidemia [30]. A debate on the effect of single exercise on blood lipids had been ensued. It has been reported that physical exercise can induce lipolysis, resulting in elevated levels of serum glycerol and higher oxidative stress in obese than in lean subjects [30]. However, no subsequent significant changes were demonstrated in the lipid profile following mild to moderateintensity exercise session which was consistent with previous findings in non-trained persons with one single session of aerobic exercise with acute [31] or moderate intensity $[32,33]$ and of similar duration. Contrastingly, a previous study illustrated the effect of exercise training on the acute response to exercise [34] and reported increase in LDL-C after acute exercise, an effect which was nullified by exercise training.
These findings propose that the magnitude of a single exercise session may have been below the required caloric threshold to induce beneficial responses in the current population. However, for acute exercise, a greater caloric expenditure should be taken with caution considering the nature of patients in this study that may be associated with a negative effect on their disease status. An earlier study was specifically designed to detect "How much exercise is enough?" and "What is the optimal amount of exercise?" with respect to potentially beneficial cardiovascular effects and effects on lipid profile and had reported that exercise has to be weekly with high amount in order to produce the acknowledged broad beneficial influences on the lipoprotein profile. The improvements were related to the activity amount and not to the exercise intensity or fitness improvement [35].

Exercise is broadly considered a cornerstone treatment for obesity and an important one in management of OA. Its influence on blood glucose is of particular interest since insulin resistance is likely to associate obesity and is reflected on the whole body systems including joints as well as the general health compromising the patient's ability to mobilize. It was proved that a single session of vigorous exercise could significantly improve insulin sensitivity measured after $24 \mathrm{~h}$ in obese adults [36]. Clearly, this vigorous exercise level cannot be prescribed for most obese OA people as a viable exercise; until now, little is understood about the influence of a lower exercise stimulus (lower duration and intensity) on sensitivity to insulin in obesity. Inconsistent results have been yielded by very few studies that have tried to investigate the metabolic benefit of shorter exercise and/or less intense sessions in obese subjects $[37,38]$. More recently, Newsom et al. demonstrated that one session of exercise with low intensity is enough to enhance insulin sensitivity into the next $24 \mathrm{~h}$ in obese adults [39] which was in line with our findings. In contrast to the popular belief, a lot of the insulin-sensitizing effect of physical exercises can be attributable to the most recent session(s) of exercises rather than to an accumulated effect of training and/or "fitness" [40, 41]. Typically, this beneficial effect of one exercise session is short-lived (i.e., 24-48 h) [42, 43]. For these reasons, exercise prescriptions should be tailored to maximize the beneficial

Table 5 Changes in glutathione reductase levels before and after exercise

\begin{tabular}{|c|c|c|c|c|c|c|c|c|}
\hline \multicolumn{9}{|l|}{ GR activity } \\
\hline \multirow{2}{*}{$\begin{array}{l}\text { \% change in score, } \\
\text { median (range) }\end{array}$} & \multicolumn{4}{|c|}{ Change between baseline and post-exercise } & \multicolumn{4}{|c|}{ Change between baseline and 24-h post-exercise } \\
\hline & Pre-exercise baseline & Post-exercise & $95 \% \mathrm{Cl}$ & $p$ value & Pre-exercise baseline & 24-h post-exercise & $95 \% \mathrm{Cl}$ & $p$ value \\
\hline $27.27(-21.3,94.6)$ & $47.92 \pm 9.75$ & $58.46 \pm 9.27$ & $(-17.92,-3.16)$ & 0.009 & $47.92 \pm 9.75$ & $49.31 \pm 9.17$ & $(-1.66,0.58)$ & 0.316 \\
\hline
\end{tabular}

Data are expressed as mean values \pm standard deviation. Parameters failing normal distribution () are shown as median value and range (in brackets). Significance level of $p$ value is $\leq 0.05$

GR glutathione reductase 
Table 6 Laboratory parameters before and after exercise

\begin{tabular}{|c|c|c|c|c|c|}
\hline & Pre-exercise baseline & Post-exercise & 24-h post-exercise & $F$ & $p$ value \\
\hline $\mathrm{TC}(\mathrm{mg} / \mathrm{dL})$ & $210 \pm 22.17$ & $222 \pm 25.37$ & $217 \pm 20.5$ & & $N / S$ \\
\hline $\mathrm{HDL}-\mathrm{C}(\mathrm{mg} / \mathrm{dL})$ & $35.71 \pm 6.3$ & $33.44 \pm 5.73$ & $35.51 \pm 3.82$ & & $\mathrm{~N} / \mathrm{S}$ \\
\hline $\mathrm{LDL}-\mathrm{C}(\mathrm{mg} / \mathrm{dL})$ & $149.71 \pm 23.34$ & $154.26 \pm 27.45$ & $152 \pm 43.28$ & & $N / S$ \\
\hline $\mathrm{TG}(\mathrm{mg} / \mathrm{dL})$ & $123.86 \pm 17.4$ & $126 \pm 19.74$ & $125 \pm 23.93$ & & $N / S$ \\
\hline IL-6 (pg/mL) & $58.46 \pm 6.51$ & $59.51 \pm 6.32$ & $57.8 \pm 6.11$ & 3.88 & 0.22 \\
\hline Glucose level & $173.92 \pm 6.24$ & $144.15 \pm 8.62$ & $168.1 \pm 6.71$ & 21.4 & 0.045 \\
\hline $\mathrm{CRP}(\mathrm{mg} / \mathrm{L})$ & $5.24 \pm 0.23$ & $5.61 \pm 0.34$ & $4.91 \pm 0.45$ & & $\mathrm{~N} / \mathrm{S}$ \\
\hline GR activity (IU/L) & $47.92 \pm 9.75$ & $58.46 \pm 9.27$ & $49.31 \pm 9.17$ & 44.73 & 0.022 \\
\hline
\end{tabular}

The intra-group variability is determined by ANOVA test. Significance level of $p$ value is $\leq 0.05$

$T C$ total cholesterol, $H D L$ high density lipoprotein, $L D L$ low density lipoprotein, $T G$ triglycerides, $I L-6$ interleukin-6, GR glutathione reductase

effects that occur in the few hours following each session of exercise. Our study has demonstrated that even a single session of low to moderate intense exercise can significantly induce a favorable effect on blood glucose level in obese OA individuals.

This study was among the scarcity to study the impact of single session of mild to moderate exercise on the obese KOA population that responds by activating antioxidant reserves. We implemented a non-exercising control group to which we compared the resting levels in KOA group. Then, improvement of participants was compared against their baseline survey findings. However, there are numerous limitations of this study. One of them was its small sample size. Therefore, the results of current study may need to be reinforced by further studies with larger numbers to be clinically relevant and could be generalized. This attenuation rate may be attributed to the difficulty in encouraging individuals with non-mild OA to commit to perform exercise. Another limitation was that the study did not examine the extended effect of exercise (i.e., for several days after exercise) and also for serial measurement during the day after exercise. A question arises in relation to the peak of exercise-induced changes and the time it fades. In the current study, we aimed to investigate the effect of single exercise on severe OA who cannot compel with frequent exercising; hence, we could not clarify the effect of KOA according to its severity on the outcomes of exercise session since we did not enroll different OA grades.

\section{Conclusion}

Symptoms of OA can be improved by mild to moderate exercise, even with a single session. Single bout of moderate exercise can enhance the anti-oxidant status and glucose tolerance of obese OA patients without provoking hazardous (undue) pro-inflammation. Obese individuals with OA should be encouraged to exercise whenever they can and to the level of intensity they can tolerate since it may be difficult to motivate them to continue regular exercise.

\section{Recommendation}

Future studies are needed on large number of patients of KOA with different grades to assess the exact role of single bout of exercise on KOA.

\section{Abbreviations}

ACR: American College of Rheumatology; BMI: Body mass index; CRP: C reactive protein; GR: Glutathione reductase; GSSG: Glutathione disulfide; HDL: High density lipoprotein; IL-6: Interleukin-6; KL: Kellgren and Lawrence; KOA: Knee osteoarthritis; LDL: Low density lipoprotein; METs: Metabolic Equivalents; MPH: Meter per hour; NADH: Nicotinamide adenine dinucleotide; ROS: Reactive oxygen species; RPE: Rate of Perceived Exertion; TC: Total cholesterol; TG: Triglycerides; VAS: Visual analogue scale; WOMAC: Western Ontario and McMaster Universities Arthritis Index

\section{Acknowledgements}

All authors wish to express great appreciations for all cooperative patients included in this study.

\section{Authors' contributions}

$-\mathrm{RS}$, TE, and SM were responsible for the idea, study design, patient selection, examination, and assessment of clinical scores. -RM and AE were responsible for laboratory analysis. -All authors were responsible for interpretation of statistical analysis and the results, writing, revising, and approval of the final submitted manuscript.

\section{Funding}

This study had no funding from any resource.

\section{Availability of data and materials}

All data are available on reasonable request.

\section{Ethics approval and consent to participate}

The present study was conducted in agreement with the guidelines of the Declaration of Helsinki. Written informed consent was received from every patient and healthy subject before participating in the study. This study was approved by the Institutional Research Board of the Faculty of Medicine, Mansoura University code R.18.04.137, date 18 April 2019.

\section{Consent for publication}

Not applicable

\section{Competing interests}

Authors declare no conflict of interest.

\section{Author details}

${ }^{1}$ Faculty of Medicine, Department of Rheumatology and Rehabilitation, Mansoura University, Elgomhoria St, Mansoura, Egypt. 'Faculty of Medicine, Department of Medical Biochemistry, Mansoura University, Mansoura, Egypt. ${ }^{3}$ Faculty of Medicine, King Khaled University, Abha, Saudi Arabia. 
Received: 2 April 2020 Accepted: 2 July 2020

Published online: 30 November 2020

\section{References}

1. Wang X, Hunter D, Xu J, Ding C (2015) Metabolic triggered inflammation in osteoarthritis. Osteoarthritis and Cartilage 23:22-30

2. Germanou El, Chatzinikolaou A, Malliou P, Beneka A, Jamurtas AZ, Bikos C, Tsoukas D, Theodorou A, Katrabasas I, Margonis K, Douroudos I, Gioftsidou A, Fatouros IG (2013) Oxidative stress and inflammatory responses following an acute bout of isokinetic exercise in obese women with knee osteoarthritis. The Knee 20:581-590

3. Mobasheri A., Biesalski H. K., Shakibaei M., Henrotin Y. Antioxidants and osteoarthritis. In: Systems biology of free radicals and antioxidants. Laher, Ismail (Ed.)Springer-Verlag Berlin Heidelberg 2014 pp 2997-3026

4. Kerksick1 C and Willoughby D. The antioxidant role of glutathione and Nacetyl-cysteine supplements and exercise-induced oxidative stress. Journal of the International Society of Sports Nutrition 2005; 2(2): 38-44.

5. Viguie CA, Frei B, Shigenaga MK et al (1993) Antioxidant status and indexes of oxidative stress during consecutive days of exercise. J Appl Physiol 75: 566-572

6. Laires MJ, Madeira F, Sergio J (1993) Preliminary study of the relationship between plasma and erythrocyte magnesium variations and some circulating pro-oxidant and antioxidant indices in a standardized physical effort. Magnesium Research 6:233-238

7. Zanuso S, Jimenez A, Pugliese G, Corigliano G, Balducci S (2010) Exercise for the management of type 2 diabetes: a review of the evidence. Acta Diabetologica 47(1):15-22

8. Golbidi S, Badran M, Laher I. Antioxidant and anti-inflammatory effects of exercise in diabetic patients. Exp Diabetes Res 2012;(Article ID 941868)

9. Fatouros IG, Jamurtas AZ (2016) Insights into the molecular etiology of exercise induced inflammation: opportunities for optimizing performance. J. Inflamm. Res. 9:175-186

10. Fatouros IG, Jamurtas AZ, Villiotou V, Pouliopoulou S, Fotinakis P, Taxildaris $K$, Deliconstantinos G (2004) Oxidative stress responses in older men during endurance training and detraining. Med. Sci. Sports Exerc. 36:2065-2072

11. Miyazaki H, Oh-ishi S, Ookawara T, Kizaki T, Toshinai K, Ha S, Haga S, Ji LL, Ohno H (2001) Strenuous endurance training in humans reduces oxidative stress following exhausting exercise. Eur. J. Appl. Physiol. 84:1-6

12. Altman R, Asch E, Bloch D, Bole G, Borenstein D, Brandt K et al (1986) Development of criteria for the classification and reporting of osteoarthritis. Classification of osteoarthritis of the knee. Diagnostic and therapeutic criteria committee of the American Rheumatism Association. Arthritis Rheum 29(8):1039-1049

13. Bellamy N, Campbell J, Stevens J, Pilcher L, Stewart C and Mahmood Z: Validation study of a computerized version of the Western Ontario and McMaster Universities VA3.0 Osteoarthritis Index J Rheumatol. 1997:24:2413.

14. Kellgren JH, Lawrence JS (1957) Radiological assessment of osteo-arthrosis. Ann Rheum Dis 16(4):494-502

15. Haskell WL, Lee IM, Pate RR, Powell KE, Blair SN et al (2007) Physical activity and public health: updated recommendation for adults from the American College of Sports Medicine and the American Heart Association. Med Sci Sports Exerc 39:1423-1434

16. Ainsworth BE, Haskell WL, Whitt MC, Irwin ML, Swartz AM et al (2000) Compendium of physical activities: an update of activity codes and MET intensities. Med Sci Sports Exerc 32:S498-S516

17. Silvis M, Sylvester J, Hacken B, Wawrzyniak J, Kelly R et al (2016) Comparison of three exercise modalities on patient reported symptoms of knee osteoarthritis: a randomized prospective study. J Arthritis 5(5):220

18. Carlberg I, Mannervik B (1985) Glutathione reductase. Methods Enzymol. 113:484-490

19. "Bosomworth NJ. Exercise and knee osteoarthritis: benefit or hazard? Can Fam Physician 2009;5599):871-8."

20. Shih M, Hootman JM, Kruger J, Helmick CG. Physical activity in men and women20. with arthritis: National Health Interview Survey, 2002. Am J Prev Med 2006;30: 21.-93.

21. Arthritis related statistics. http://www.cdc.gov/arthritis/data_statistics/ arthritis_related_stats.htm; 2006.

22. Blagojevic M, Jinks C, Jeffery A, Jordan KP (2010) Risk factors for onset of osteoarthritis of 22. the knee in older adults: a systematic review and metaanalysis. Osteoarthritis Cartilage 18:24-33
23. Sowers MF, Yosef M, Jamadar D, Jacobson J, Karvonen-Gutierrez C, Jaffe M (2008) BMI vs. body composition and radiographically defined osteoarthritis of the knee in women: a 4-year follow-up study. Osteoarthritis Cartilage 16: 367-372

24. Radak Z, Zhao Z, Koltai E, Ohno H, Atalay M (2013) Oxygen consumption and usage during physical exercise: the balance between oxidative stress and ROS-dependent adaptive signaling. Antioxidants Redox Signaling. 18: 1208-1246

25. Powers SK, Nelson WB, Hudson MB. Exercise-induced oxidative stress in humans: cause and consequences.Free Radic Biol Med. 2011; 51: 942-950.

26. Margaritelis NV, Kyparos A, Paschalis V, Theodorou AA, Panayiotou G, Zafeiridis A et al (2014) Reductive stress after exercise: the issue of redox individuality. Redox Biol. 2:520-528

27. Huang CJ, McAllister MJ, Slusher AL, Webb HE, Mock JT, Avecedo EO (2015) Obesity-related oxidative stress: the impact of physical activity and diet manipulation. Sports Med Open. 1:32

28. Accattato F, Greco M, Pullano SA, Carè I, Fiorillo AS, Pujia A et al (2017) Effects of acute physical exercise on oxidative stress and inflammatory status in young, sedentary obese subjects. PLoS ONE 12(6):e0178900

29. "Hunter DJ, Eckstein F. Exercise and osteoarthritis" J Anat 2009; 214(2): 197207.

30. Lim HJ (2006) The effects of aerobic exercise program on body composition and blood lipids in obesity high school girls. J Kor Soc Living Environ System 13:189-194

31. Petridou A, Mougios V (2002) Acute changes in triacylglycerol lipase activity of human adipose tissue during exercise. J Lipid Res. 43:1331-1334

32. Greene N.P., Martin S.E., Crouse S.F.. Acute exercise and training alter blood32. lipid and lipoprotein profiles differently in overweight and obese men and women. Obesity 2012;20, 1618-1627)

33. Lahiji F, Vasaghi Gharamaleki B, Mirzaii Dizgah I, Abdollahi A, Attarbashi MB et al (2015) The effect of a single session of aerobic or resistance exercise on salivary lipid profile. J Arch Mil Med. 3(1):e26341

34. Pronk NP, Crouse SF, O'Brien BC, Rohack JJ (1995) Acute effects of walkingon serum lipids and lipoproteins in women. J Sports Med Phys Fitness 35:50-58

35. Crouse SF, O'Brien BC, Grandjean PW et al (1997) Effects of training and a singlesession of exercise on lipids and apolipoproteins in hypercholesterolemicmen. J Appl Physiol 83:2019-2028

36. K Raus W.E., Houmard J.A., Duscha B.D., Knetzger K.J., Wharton M.B., J. S. Mccartney, C.W. Bales, S.Henes,G.P. Samsa, J.D. Otvos, K.R. Kulkarni, C.A. Slentz. Effects of the amount and intensity of exercise on plasma lipoproteins. N Engl J Med 2002;347:1483-1492.

37. Devlin JT, Horton ES (1985) Effects of prior high-intensity exercise on glucose metabolism in normal and insulin-resistant men. Diabetes 34:973979

38. Manders RJ, Van Dijk JW, van Loon LJ (2010) Low-intensity exercise reduces the prevalence of hyperglycemia in type 2 diabetes. Med Sci Sports Exerc 42:219-225

39. Zhang JQ, Ji LL, Fretwell VS, Nunez G (2006) Effect of exercise on postprandial lipemia in men with hypertriglyceridemia. Eur J Appl Physiol 98:575-582

40. Newsom SA, Hinko A, Everett AC, Horowitz JF (2013) A single session of low-intensity exercise is sufficient to enhance insulin sensitivity into the next day in obese adults. Diabetes Care 36:2516-2522

41. Heath GW, Gavin JR 3rd, Hinderliter JM, Hagberg JM, Bloomfield SA, Holloszy JO (1983) Effects of exercise and lack of exercise on glucose tolerance and insulin sensitivity. J Appl Physiol 55:512-517

42. King DS, Dalsky GP, Clutter WE et al (1988) Effects of exercise and lack of exercise on insulin sensitivity and responsiveness. J Appl Physiol 64:19421946

43. Dela F, Mikines KJ, von Linstow M, Secher NH, Galbo H (1992) Effect of training on insulin-mediated glucose uptake in human muscle. Am J Physiol 263:E1134-E1143

\section{Publisher's Note}

Springer Nature remains neutral with regard to jurisdictional claims in published maps and institutional affiliations. 